\title{
Criminologie
}

\section{Synthèse des connaissances sur les facteurs de protection liés à la délinquance}

\section{Frédéric Ouellet et Sheilagh Hodgins}

Volume 47, numéro 2, automne 2014

URI : https://id.erudit.org/iderudit/1026735ar

DOI : https://doi.org/10.7202/1026735ar

Aller au sommaire du numéro

\section{Éditeur(s)}

Les Presses de l'Université de Montréal

ISSN

0316-0041 (imprimé)

1492-1367 (numérique)

Découvrir la revue

Citer cet article

Ouellet, F. \& Hodgins, S. (2014). Synthèse des connaissances sur les facteurs de protection liés à la délinquance. Criminologie, 47(2), 231-262.

https://doi.org/10.7202/1026735ar
Résumé de l'article

La plupart des enfants et des adolescents qui commettent des actes délinquants ne persistent pas dans la criminalité une fois à l'âge adulte. Ainsi, on conçoit qu'il y a dans l'environnement immédiat des facteurs qui protègent les enfants et les adolescents à risque. Or, la documentation sur les facteurs de protection montre des lacunes. Notamment, très peu d'études nous renseignent sur les fondements conceptuels et méthodologiques qui encadrent l'examen de ces facteurs. Cet article synthétise les connaissances sur la base de travaux empiriques sur les facteurs de protection associés aux comportements antisociaux et criminels et propose d'examiner les considérations conceptuelles, méthodologiques et les limites des études sélectionnées susceptibles d'influencer les facteurs découverts. Les résultats montrent qu'un grand nombre de facteurs de protection ont été identifiés. Le manque d'uniformité dans les résultats reflète le caractère exploratoire des études, mais aussi la quasi-absence de validation des facteurs découverts. Parmi les questions méthodologiques abordées, l'importance des caractéristiques de la population est mise en évidence. Sur la base de nos résultats, des pistes de recherche sont proposées, notamment de considérer l'hétérogénéité des populations qui affichent des risques élevés de criminalité à l'âge adulte. Les connaissances sur des facteurs de protection sont nécessaires pour éclairer les décisions politiques concernant l'investissement dans les programmes visant à prévenir la criminalité dès la petite enfance.
Ce document est protégé par la loi sur le droit d'auteur. L’utilisation des services d'Érudit (y compris la reproduction) est assujettie à sa politique d'utilisation que vous pouvez consulter en ligne.

https://apropos.erudit.org/fr/usagers/politique-dutilisation/ 


\title{
Synthèse des connaissances sur les facteurs de protection liés à la délinquance ${ }^{1}$
}

\author{
Frédéric Ouellet ${ }^{2}$ \\ Professeur adjoint \\ École de criminologie, Université de Montréal \\ frederic.ouellet.1@umontreal.ca \\ Sheilagh Hodgins \\ Professeure associée \\ Département de psychiatrie, Université de Montréal \\ s.hodgins@umontreal.ca
}

RÉSUMÉ - La plupart des enfants et des adolescents qui commettent des actes délinquants ne persistent pas dans la criminalité une fois à l'âge adulte. Ainsi, on conçoit qu'il y a dans l'environnement immédiat des facteurs qui protègent les enfants et les adolescents à risque. Or, la documentation sur les facteurs de protection montre des lacunes. Notamment, très peu d'études nous renseignent sur les fondements conceptuels et méthodologiques qui encadrent l'examen de ces facteurs. Cet article synthétise les connaissances sur la base de travaux empiriques sur les facteurs de protection associés aux comportements antisociaux et criminels et propose d'examiner les considérations conceptuelles, méthodologiques et les limites des études sélectionnées susceptibles d'influencer les facteurs découverts. Les résultats montrent qu'un grand nombre de facteurs de protection ont été identifiés. Le manque d'uniformité dans les résultats reflète le caractère exploratoire des études, mais aussi la quasi-absence de validation des facteurs découverts. Parmi les questions méthodologiques abordées, l'importance des caractéristiques de la population est mise en évidence. Sur la base de nos résultats, des pistes de recherche sont proposées, notamment de considérer l'hétérogénéité des populations qui affichent des risques élevés de criminalité à l'âge adulte. Les connaissances sur des facteurs de protection sont nécessaires pour éclairer les décisions

1. Cette synthèse des connaissances a été réalisée grâce à des fonds du Centre national de prévention du crime du Canada (CNPC); un merci spécial à Annie K. Yessine du CNPC pour ses commentaires.

2. Université de Montréal, École de criminologie, Pavillon Lionel-Groulx, C. P. 6128, succursale Centre-ville, Montréal (Québec), Canada, H3C 3J7. 
politiques concernant l'investissement dans les programmes visant à prévenir la criminalité dès la petite enfance.

MOTS CLÉS • Facteurs de protection, délinquance, comportements antisociaux, synthèse des connaissances.

\section{Introduction}

L'engagement dans la délinquance ou la criminalité ne relève pas du simple hasard, certains individus sont davantage à risque de se lancer dans de telles trajectoires. Il devient alors prioritaire de cibler ces individus qui s'engagent et persistent dans la délinquance, puisque ce groupe de délinquants «chroniques» serait responsable de près de $50 \%$ à $70 \%$ des crimes qui sont commis dans nos sociétés occidentales (Blumstein, Cohen \& Farrington, 1988; Chaiken \& Chaiken, 1982; Kratzer \& Hodgins, 1999; Moffitt, Caspi, Harrington \& Milne, 2002; Piquero, 2000; Pulkkinen, 1998; Wilkstrom, 1985; Wolfgang, Figlio \& Sellin, 1972). Ceci explique, du moins en partie, l'importance du corpus de travaux consacré à l'identification des facteurs de risque liés à la commission d'actes criminels (Farrington \& Ttofi, 2011). On comprend bien que les facteurs de risque sont importants; toutefois, à l'égard du développement de la délinquance et des comportements antisociaux, une approche centrée uniquement sur les facteurs de risque laisse certaines questions fondamentales en suspens.

Bien que les recherches longitudinales montrent que la délinquance juvénile et la criminalité à l'âge adulte sont l'aboutissement d'un long processus de développement, on connaît encore peu la constellation de facteurs qui permet d'expliquer pourquoi certains individus à risque à l'enfance ou à l'adolescence ne s'impliquent tout simplement jamais ou se désistent du crime une fois à l'âge adulte. Cette question est importante puisque parmi les enfants et les adolescents qui présentent des facteurs de risque, peu persistent dans le crime une fois adulte. On conçoit qu'il y a dans l'environnement immédiat des facteurs qui protègent la majorité de ces enfants/adolescents. Dans la littérature, de multiples explications biologiques, sociologiques et psychologiques ont été proposées, mais leurs fondements empiriques demeurent minces (Stouthamer-Loeber, Wei, Loeber \& Masten, 2004). En 2003, Lösel et Bender qualifiaient l'état des connaissances sur les facteurs de protection de rudimentaire et la situation a peu avancé depuis (voir aussi Hall et al., 2012b). 
En raison du potentiel de ces facteurs, tant sur le plan de l'intervention clinique que de la gestion du risque, nous proposons dans cet article une synthèse critique des connaissances sur les facteurs de protection. En particulier, l'identification de facteurs de protection «naturels» pourrait notamment servir à orienter les programmes de prévention dès l'enfance et à formuler des réponses adaptées aux enfants et aux adolescents à risque. Pour Hurrelmann et Lösel (1990), l'étude à la fois des sources de vulnérabilité et de résilience représente un pan prometteur de la recherche vers une meilleure prévention, afin de réduire l'impact des facteurs de risque, tout en améliorant la résistance des individus.

L'état des connaissances et le contexte derrière l'étude des facteurs de protection

D'abord, au regard des conduites délinquantes et antisociales, comment expliquer l'écart des connaissances entre les facteurs de risques et les facteurs de protection? Par exemple, sur le développement de la délinquance, les modèles théoriques existants sont davantage axés sur les facteurs de risque individuels et environnementaux (pour une revue plus complète de ces facteurs de risque, voir Loeber, 1990; Loeber \& Farrington, 2000; Farrington \& Welsh, 2007) qu'ils le sont sur les facteurs qui préviennent ou qui réduisent les risques qu'un individu entreprenne une carrière criminelle.

Ensuite, Stattin et ses collègues (1997) montrent que les précurseurs négatifs ou indésirables qui se lient à la délinquance ont tendance à faire davantage consensus dans les modèles criminologiques, alors que la même entente n'est pas aussi manifeste pour les facteurs dont l'effet est positif. Farrington (2000) soutient également que la conceptualisation des facteurs de protection varie passablement entre les études et que celle-ci est d'ailleurs une source de mésentente entre les chercheurs.

Il est difficile d'arriver à une définition conceptuelle commune pour plusieurs raisons. La diversité des objets d'étude auxquels on associe les facteurs de protection en est une. Le spectre des conduites délinquantes et antisociales est large, ce qui ajoute à la complexité du phénomène et à la difficulté de limiter la définition conceptuelle à un type particulier de conduites. De plus, dans ce champ large de la délinquance et des comportements antisociaux, il est possible de s'intéresser autant aux facteurs qui prédisent l'absence d'une initiation à la criminalité qu'à 
ceux liés au processus de désistement. Il est également difficile d'arriver à une définition conceptuelle commune car dans certains cas on considère les facteurs de protection comme l'envers des facteurs de risque et dans d'autres comme des facteurs distincts. À l'origine, il était usuel de concevoir les facteurs de protection comme l'opposé ou le contraire des facteurs de risque (Masten \& Garmezy, 1985). Le spectre des facteurs s'est depuis élargi. Les travaux de Rutter $(1985,1987)$ ont permis de rendre compte de la complexité qui entoure la conceptualisation des facteurs de protection et, par extension, d'accroître les horizons dans lesquels chercher des facteurs potentiels.

L'absence de consensus sur le plan conceptuel est exacerbée par la faible quantité de travaux centrés sur les facteurs qui préviennent les délinquances et autres conduites antisociales. La rareté des données prospectives explique le peu d'antécédents empiriques. Les grandes études longitudinales prospectives ont rarement modélisé ou théorisé les facteurs de protection a priori, ces enquêtes étaient initialement pensées pour étudier les facteurs de risque. Pour conclure, le manque d'uniformité conceptuelle entre les études, ajouté à une littérature peu abondante, laisse nécessairement plusieurs questions en suspens. On se questionne notamment quant à la portée et la validité des facteurs de protection découverts à ce jour.

\section{L'initiative du Centers for Disease Control and Prevention}

Les lacunes dans la littérature sur les facteurs de protection ont incité la création d'un groupe d'experts intéressés à l'examen et à la progression de la recherche sur les facteurs qui préviennent la perpétration de violences graves chez les jeunes. Les résultats de cette initiative du Centers for Disease Control and Prevention ont été publiés dans un numéro spécial de l'American Journal of Preventive Medecine paru en août $2012^{3}$. Dans le cadre de cet atelier, les experts se sont concertés sur la définition, ainsi que la démarche méthodologique à adopter. Les facteurs de protection ont été définis comme des facteurs qui précèdent la perpétration de la violence des jeunes et qui prédisent une faible probabilité de passage à l'acte violent des jeunes dans la population générale (Hall et al., 2012b, S3). Dans leur article, Lösel et Farrington (2012) passent en revue les facteurs susceptibles de prévenir les actes

3. Quatre des études publiées dans ce numéro sont incluses dans notre synthèse des résultats. 
de violence graves perpétrés par les jeunes. La grande contribution de ce numéro spécial de l'American Journal of Preventive Medicine, consacré à l'étude des facteurs de protection, est d'avoir fourni, dans la mesure du possible, un cadre conceptuel commun aux recherches incluses dans le numéro.

Cet exercice fort instructif a entre autres permis de constater qu'en dépit d'un cadre conceptuel s'apparentant d'une étude à l'autre, les facteurs de protection varient passablement entre les études). Hall et ses collègues (2012a, S80) concluent que les facteurs peuvent avoir différents effets selon le contexte qui entoure l'étude, en particulier ils établissent la provenance de l'échantillon comme une cause probante de ces variations. Ils reconnaissent que ces influences contextuelles méritent d'être examinées afin de mieux connaître comment elles opèrent.

\section{La présente étude}

Dans le numéro spécial de l'American Journal of Preventive Medecine, Lösel et Farrrington (2012) se fondent sur un nombre important de travaux pour formuler et élaborer leur cadre conceptuel, le spectre des objets d'étude y est relativement large, p. ex: la violence chez les jeunes, les facteurs de risque et de protection, ainsi que bon nombre d'études qui portent sur des questions méthodologiques. À la différence de cet article, la synthèse des connaissances présentée dans cette étude se veut plus ciblée: un regard plus approfondi sur les études portant sur les facteurs qui préviennent les conduites délinquantes et antisociales y est proposé. Cet article présente une synthèse critique des connaissances qui s'inscrit dans la foulée des travaux sur le développement de la délinquance et de la criminalité. L'examen repose ainsi sur un nombre de travaux restreints sélectionnés selon des critères préalablement établis. L'objectif est de faire ressortir les différents facteurs de protection identifiés, pour ensuite analyser l'impact des considérations conceptuelles et méthodologiques sur ceux-ci. L'idée derrière l'analyse présentée est de fournir des pistes utiles pour l'orientation et le développement de projets de recherche sur les facteurs de protection. Finalement, nous ferons un retour sur les éléments soulevés par Lösel et Farrington. 


\section{Méthodologie}

Avant d'examiner les constats qui émanent de cette synthèse critique des connaissances, il importe de détailler la démarche méthodologique mise en œuvre: les stratégies déployées dans la recherche de travaux empiriques, aussi la procédure et les critères de sélection des études.

\section{Démarche de la synthèse critique des connaissances}

Voici la description des différentes étapes de cette démarche, celle-ci est nécessaire afin de détailler les ressources consultées et les critères d'admissibilité privilégiés dans le choix des études.

\section{Procédure de recherche}

La recherche de travaux empiriques, en anglais et en français, sur les facteurs de protection ne s'est pas limitée à une période précise. Le nombre restreint de travaux sur la question justifie cette décision. Précisons toutefois que cette recension des écrits s'est terminée en avril 2014. Essentiellement, trois procédures ont été mises de l'avant pour trouver des études pertinentes. La première consistait, à l'aide de nombreux mots-clés, à consulter différentes ressources électroniques. Les ressources consultées (outils de recherche et banque de données) sont les suivantes: Google Scholar, Érudit, Taylor \& Francis, PsycInfo, Medline et PubCrawler. La liste des mots-clés utilisés est présentée dans le tableau 1. L'utilisation des facteurs de protection ne se limite pas aux études en criminologie; ainsi, pour orienter notre recherche, plusieurs combinaisons de mots-clés ont été effectuées, notamment entre les mots-clés de la première colonne et ceux de la deuxième. La deuxième méthode de recherche a été de procéder par «boule de neige». Autrement dit, nous avons fait le suivi des références des études pertinentes que nous avons trouvées. Finalement, la troisième procédure visait à examiner les textes ayant cité les études ciblées par les deux premiers moyens mis de l'avant. Cette troisième méthode nous a permis d'accéder aux recherches les plus récentes dans ce domaine d'étude. Autrement dit, nous avons utilisé les articles découverts par le biais de la première et de la deuxième procédure de recherche comme source d'identification d'études additionnelles. 
TAB LEA U 1

Liste des mots-clés

\begin{tabular}{|l|l|}
\hline \multicolumn{2}{|c|}{ Mots-clés } \\
\hline $\begin{array}{l}\text { Facteurs de protection (Protective } \\
\text { factors) }\end{array}$ & $\begin{array}{l}\text { Comportements antisociaux (Antisocial } \\
\text { behaviour) }\end{array}$ \\
\hline Promotive factors & Délinquance (Delinquency) \\
\hline Protection (Protection) & Criminalité (Criminality) \\
\hline Désistement (Desistance) & Passage à l'acte criminel (Offending) \\
\hline Résilience (Resilience) & $\begin{array}{l}\text { Troubles/problèmes de conduite } \\
\text { (Conduct problem) }\end{array}$ \\
\hline $\begin{array}{l}\text { Ressources personnelles (Personal } \\
\text { ressources) }\end{array}$ & \\
\hline
\end{tabular}

Critères d'admissibilité des études

La procédure mise de l'avant a donné lieu à une recherche exhaustive qui a généré une quantité importante d'articles, 66 études pour être plus exact. Afin d'atteindre les objectifs fixés au préalable, des critères d'admissibilité ont été appliqués. En somme, pour qu'une étude soit retenue, elle devait se conformer à un certain nombre de critères. Premièrement, il devait s'agir de travaux empiriques intéressés à l'identification/validation et à l'effet de facteurs qui préviennent les conduites délinquantes, criminelles ou antisociales. Deuxièmement, seuls les écrits scientifiques ont été retenus. Les études proviennent essentiellement de revues avec comité de lecture $(\mathrm{N}=19)$, deux chapitres de livre ont également été admis. Des problèmes de rigueur méthodologique sont plus susceptibles d'affliger les synthèses dans lesquelles une grande partie de l'échantillon des études retenues comprend des recherches non publiées ou des études publiées dans des revues sans comité de lecture où la qualité de ces travaux est inégale et possiblement douteuse dans certains cas (Pratt, 2002). Troisièmement, les recherches incluses se devaient également de montrer une démarche méthodologique détaillée et rigoureuse; le devis méthodologique se devait d'être reproductible. Lorsque la synthèse des connaissances est basée sur un échantillon d'études qui présentent des problèmes sur le plan méthodologique, les conclusions tirées de cette synthèse sont par conséquent suspectes (Rosenthal \& DiMatteo, 2001).

L'application de ces critères sur les 66 études préalablement identifiées a été effectuée par une seule personne. Toutefois, il importe de 
préciser qu'une autre personne s'est impliquée dans le processus d'élimination (un professeur-chercheur de la même institution, mais d'un autre département), avant d'écarter de manière définitive les manuscrits, cette personne s'est affairée à valider les décisions prises. Il faut souligner que les deux personnes engagées dans le processus d'élimination sont arrivées aux mêmes conclusions. La sélection des études se base sur des résumés de lecture. Ces résumés mettent en évidence les principaux éléments de la problématique, les objectifs, la méthodologie, les résultats, les conclusions et des limites de l'étude. Au final, 21 études sont intégrées dans cette synthèse des connaissances. Le tableau 2 renseigne sur les études retenues et leurs principales caractéristiques.

L'intérêt récent pour les facteurs de protection est mis en évidence dans cette synthèse, la plus ancienne étude retenue date de 1994. L'ensemble des études examinées ici $(\mathrm{N}=21)$ se base, en partie ou en totalité, sur des données issues d'enquêtes longitudinales ou rétrospectives. Les fondements biographiques derrière les facteurs de protection rendent nécessaire l'utilisation de telles données. L'étude de phénomène qui implique le passage de l'état d'origine à l'état de destination, par exemple le passage à l'acte criminel à l'âge adulte, ne peut être réalisée convenablement que si l'on dispose de la biographie complète de chaque répondant (Laplante, Street \& Santillan, 2006). Les biographies individuelles sont rarement à la disposition des chercheurs, mais certaines méthodes alternatives permettent de pallier cette limite. Deux méthodes sont utilisées pour recueillir ce type de données. La première se fonde sur un questionnaire biographique rétrospectif qui couvre une longue période lors du premier et seul passage (A, B, L, P, Q, R, T). La seconde se base également sur un questionnaire biographique rétrospectif, à la différence que dans ce cas-ci, le passage du questionnaire se répète dans le temps (p. ex. chaque année), ainsi les périodes couvertes sont plus courtes et portent seulement sur la période écoulée entre le dernier passage et le passage présent (C, D, E, F, G, H, I, J, K, N, M, O, S, U). Bien que ces deux méthodes soient utilisées dans la recherche sur les facteurs de protection, on conçoit que les données d'études longitudinales prospectives (la deuxième méthode décrite) offrent un portrait plus exact (Hall et al., 2012b).

La symétrie entre les études au regard du type de données s'avère toutefois une exception lorsque l'on tient compte de différentes considérations méthodologiques. Par exemple, qu'il s'agisse de la taille de la population ou encore des caractéristiques des participants à l'examen, 
nous constatons de nombreuses disparités entre les études. Nous désirons dans cette synthèse porter attention à ces disparités et à leur impact.

\section{Stratégie d'analyse}

L'analyse descriptive et la présentation tabulaire des données extraites des études sélectionnées sont issues d'une synthèse qualitative. Comme il vient d'être exposé, la grande variabilité entre les études, tant sur le plan méthodologique que des résultats obtenus, justifie une synthèse de nature qualitative, en opposition à une synthèse de nature quantitative (méta-analyse). Le but de l'exercice ici n'est pas de débattre de la relation entre les facteurs de protection et les conduites délinquantes et antisociales, celle-ci est bien démontrée. L'objectif consiste d'abord à relever les facteurs de protection dans les travaux sélectionnés et ensuite d'examiner de plus près ce qui explique la disparité des facteurs découverts. 
TABLEAU 2

Les études sélectionnées et leurs principales caractéristiques

\begin{tabular}{|c|c|c|c|c|c|c|}
\hline ID & Auteurs & $\begin{array}{l}\text { Année de } \\
\text { publication }\end{array}$ & Échantillon & $\begin{array}{c}\text { Sexe des } \\
\text { participants }\end{array}$ & $\begin{array}{c}\text { Stade du } \\
\text { développement }\end{array}$ & $\begin{array}{l}\text { Comportements } \\
\text { prédits }\end{array}$ \\
\hline A & Arthur et al. & 2002 & $\begin{array}{l}\mathrm{N}=11564 \\
\text { Population générale }\end{array}$ & Mixte & Adolescence & $\begin{array}{l}\text { Délinquance et abus } \\
\text { de substance }\end{array}$ \\
\hline B & Baglivio et al. & 2014 & $\begin{array}{l}\text { N = } 34497 \\
\text { Les jeunes référés au Florida Department of } \\
\text { Juvenile Justice. Subdivise l'échantillon en } \\
3 \text { groupes de jeunes selon la délinquance } \\
\text { (sévérité, violence et chronicité) }\end{array}$ & Mixte & $\begin{array}{l}\text { Enfance (âgé de } \\
\text { moins de } \\
12 \text { ans), } \\
\text { adolescence } \\
\text { (16 ans et plus) }\end{array}$ & $\begin{array}{l}\text { Récidive officielle sur } \\
\text { une période de } \\
12 \text { mois }\end{array}$ \\
\hline C & Bushway et al. & 2013 & $\begin{array}{l}\mathrm{N}=846 \\
\text { Mesures mises en place pour rejoindre les } \\
\text { jeunes à risque (violence et délinquance } \\
\text { persistante) (Rochester Youth Development } \\
\text { Study) }\end{array}$ & Mixte & $\begin{array}{l}\text { Adolescence, } \\
\text { début de l'âge } \\
\text { adulte }\end{array}$ & $\begin{array}{l}\text { La prévalence des } \\
\text { comportements } \\
\text { violents et le port } \\
\text { d'une arme }\end{array}$ \\
\hline D & Bender \& Lösel & 1997 & $\begin{array}{l}\mathrm{N}=100 \\
\text { Logements sociaux }\end{array}$ & Mixte & Adolescence & $\begin{array}{l}\text { Conduites } \\
\text { antisociales }\end{array}$ \\
\hline $\mathbf{E}$ & Bernat et al. & 2012 & $\begin{array}{l}\mathrm{N}=1037-1226 \\
\text { Population générale (Add Health) }\end{array}$ & Mixte & $\begin{array}{l}\text { Adolescence, } \\
\text { début de l'âge } \\
\text { adulte }\end{array}$ & Actes violents \\
\hline $\mathbf{F}$ & Farrington et al. & 2008 & $\begin{array}{l}\mathrm{N}=1009 \\
\text { Population générale et jeunes antisociaux } \\
\text { (Pittsburgh Study) }\end{array}$ & Garçons & $\begin{array}{l}\text { Enfance, } \\
\text { adolescence, } \\
\text { début de l'âge } \\
\text { adulte }\end{array}$ & Actes violents et vols \\
\hline
\end{tabular}


TABLEAU 2 (suite)

\begin{tabular}{|l|l|c|l|c|l|l|}
\hline ID & \multicolumn{1}{|c|}{ Auteurs } & $\begin{array}{c}\text { Année de } \\
\text { publication }\end{array}$ & \multicolumn{1}{|c|}{ Échantillon } & $\begin{array}{c}\text { Sexe des } \\
\text { participants }\end{array}$ & $\begin{array}{c}\text { Stade du } \\
\text { développement }\end{array}$ & \multicolumn{1}{c|}{$\begin{array}{c}\text { Comportements } \\
\text { prédits }\end{array}$} \\
\hline G & $\begin{array}{l}\text { Farrington \& } \\
\text { Ttofi }\end{array}$ & 2011 & $\begin{array}{l}\mathrm{N}=411 \\
\text { Classe ouvrière (Cambridge Study) }\end{array}$ & Garçons & $\begin{array}{l}\text { Milieu-fin de } \\
\text { l'enfance }\end{array}$ & $\begin{array}{l}\text { Activités criminelles } \\
\text { à l'âge adulte }\end{array}$ \\
\hline H & Henry et al. & 2012 & $\begin{array}{l}\mathrm{N}=4432 \\
\text { Population générale }\end{array}$ & Mixte & $\begin{array}{l}\text { Début de } \\
\text { l'adolescence }\end{array}$ & Actes violents \\
\hline I & Herrenkohl et al. & 2003 & $\begin{array}{l}\mathrm{N}=808 \\
\text { Étudiants d'écoles publiques }\end{array}$ & Mixte & Adolescence & $\begin{array}{l}\text { Comportements } \\
\text { violents graves }\end{array}$ \\
\hline J & Herrenkohl et al. & 2005 & $\begin{array}{l}\mathrm{N}=416 \\
\text { Enfants exposés à des abus physiques }\end{array}$ & Mixte & Enfance & $\begin{array}{l}\text { Conduites antiso- } \\
\text { ciales }\end{array}$ \\
\hline K & Herrenkokl et al. & 2012 & $\begin{array}{l}\mathrm{N}=808 \\
\text { Étudiants d'écoles publiques }\end{array}$ & Mixte & $\begin{array}{l}\text { Fin de l'enfance, } \\
\text { adolescence }\end{array}$ & Actes violents \\
\hline L & Hoge \& Leschied & 1996 & $\begin{array}{l}\mathrm{N}=338 \\
\text { Ont au moins commis un délit }\end{array}$ & Adolescence & $\begin{array}{l}\text { Activités criminelles } \\
\text { et association aux } \\
\text { pairs délinquants }\end{array}$ \\
\hline M & Lösel \& Bender & 2014 & $\begin{array}{l}\mathrm{N}=519 \\
\text { Population générale (Erlanger-Nuremberg } \\
\text { Development and Prevention Study) }\end{array}$ & Mixte & $\begin{array}{l}\text { Fin de l'enfance, } \\
\text { début de } \\
\text { l'adolescence }\end{array}$ & $\begin{array}{l}\text { Comportements } \\
\text { antisociaux et } \\
\text { violents }\end{array}$ \\
\hline N & $\begin{array}{l}\text { Lösel \& } \\
\text { Bliesener }\end{array}$ & 1994 & $\begin{array}{l}\text { N=146 } \\
\text { Milieux problématiques }\end{array}$ & $\begin{array}{l}\text { Mixte } \\
\text { Population générale } \\
\text { (Pittsburgh Study) }\end{array}$ & Adolescence & $\begin{array}{l}\text { Problème de } \\
\text { conduites }\end{array}$ \\
\hline $\mathbf{O}$ & Pardini et al. & 2012 & Garçons & Adolescence & Actes violents \\
\hline
\end{tabular}


TABLEAU 2 (suite)

\begin{tabular}{|c|c|c|c|c|c|c|}
\hline ID & Auteurs & $\begin{array}{c}\text { Année de } \\
\text { publication }\end{array}$ & Échantillon & $\begin{array}{c}\text { Sexe des } \\
\text { participants }\end{array}$ & $\begin{array}{c}\text { Stade du } \\
\text { développement }\end{array}$ & $\begin{array}{l}\text { Comportements } \\
\text { prédits }\end{array}$ \\
\hline $\mathbf{P}$ & Rennie \& Dolan & 2010 & $\begin{array}{l}\mathrm{N}=135 \\
\text { En détention provisoire }\end{array}$ & Garçons & Adolescence & $\begin{array}{l}\text { Arrestation, activités } \\
\text { criminelles et traits } \\
\text { de personnalité } \\
\text { antisociaux }\end{array}$ \\
\hline $\mathbf{Q}$ & $\begin{array}{l}\text { Stattin, } \\
\text { Romelsjö \& } \\
\text { Stenbacka }\end{array}$ & 1997 & $\begin{array}{l}\mathrm{N}=7577 \\
\text { Lors de la conscription militaire en Suède: } \\
\text { population générale }\end{array}$ & Garçons & Âge adulte & Criminalité future \\
\hline $\mathbf{R}$ & Stoddard et al. & 2013 & $\begin{array}{l}\mathrm{N}=726 \\
\text { Échantillon sélectionné dans un département } \\
\text { d'urgence en intervention pour la consomma- } \\
\text { tion d'alcool et les comportements violents }\end{array}$ & Mixte & $\begin{array}{l}\text { Adolescence } \\
\text { (14-18 ans) }\end{array}$ & $\begin{array}{l}\text { Comportements } \\
\text { violents }\end{array}$ \\
\hline $\mathrm{S}$ & $\begin{array}{l}\text { Stouthamer- } \\
\text { Loeber et al. }\end{array}$ & 2004 & $\begin{array}{l}\mathrm{N}=506 \\
\text { Délinquance persistante à l'adolescence } \\
\text { (Pittsburgh Study) }\end{array}$ & Garçons & Adolescence & $\begin{array}{l}\text { Désistement criminel } \\
\text { à l'âge adulte }\end{array}$ \\
\hline $\mathbf{T}$ & Taylor et al. & 2004 & $\begin{array}{l}\mathrm{N}=162 \\
\text { Criminels (81) et non-criminels (81) (Wulan, } \\
\text { Chine) }\end{array}$ & $\begin{array}{l}\text { Mixte (très } \\
\text { peu de filles } \\
{[N=10] \text { ) }}\end{array}$ & Adolescence & Criminalité future \\
\hline $\mathbf{U}$ & $\begin{array}{l}\text { van Domburgh } \\
\text { et al. }\end{array}$ & 2009 & $\begin{array}{l}\mathrm{N}=310 \\
\text { Crime commis avant l'âge de } 12 \text { ans } \\
\text { (Pittsburgh Study) }\end{array}$ & Garçons & Enfance & $\begin{array}{l}\text { Délinquance à } \\
\text { l'adolescence }\end{array}$ \\
\hline
\end{tabular}




\section{Résultats}

Les facteurs de protection présentés au tableau 3 ont été identifiés comme étant des éléments qui prévenaient de manière significative les conduites délinquantes et antisociales ${ }^{4}$. Notre synthèse met en évidence la grande diversité des facteurs de protection identifiés, il est d'ailleurs possible de classifier ces facteurs selon leur nature: individuelle, familiale et sociale. À l'intérieur de ces grandes catégories, il est également possible de ranger les facteurs dans diverses thématiques. Les études retenues ont permis d'identifier en moyenne 3,65 facteurs de protection. Le tableau 3 montre aussi que la majorité des études considèrent plusieurs thématiques (1,94 en moyenne), mais aussi plusieurs catégories (2,71 en moyenne) dans l'identification des facteurs de protection. Ceci démontre bien qu'il y a de multiples sources desquelles émanent les facteurs qui protègent la majorité de ces enfants/adolescents/adultes. Bien que la majorité des études recensées accordent une importance aux différentes catégories de facteurs de protection, on constate que peu de facteurs font l'objet de validation. Les critères de sélection des facteurs ne sont pas les mêmes entre les études, ce qui rend les comparaisons difficiles et fait que l'on a peu de facteurs qui peuvent être considérés comme ayant fait l'objet d'une contre-validation empirique. Les sections suivantes tentent d'expliquer cet état de fait, notamment expliquent l'importante diversité des facteurs découverts et l'absence apparente de validation. La réponse à ces questions se niche dans différentes dimensions, une attention particulière est accordée à sept de celles-ci. Ces dimensions ont été divisées selon deux thèmes: les considérations méthodologiques et les caractéristiques des populations étudiées. Les thèmes et les dimensions sont issus d'une démarche intellectuelle, l'analyse documentaire présentée se fonde sur deux opérations: les résumés de lecture et l'indexation.

4. Les facteurs ont été identifiés à l'aide d'analyses bivariées ou multivariées. 
TA B LEA U 3

Les facteurs de protection identifiés dans les études retenues par thématiques et catégories

\begin{tabular}{|c|}
\hline Facteurs individuels \\
\hline Traits de personnalité/tempérament \\
\hline Traits de la personnalité résiliente $(P)$ \\
\hline Faible anxiété $(F ; M)$ \\
\hline Peu de traits psychopathiques (F) \\
\hline $\begin{array}{l}\text { Peu de symptômes du trouble du déficit d'attention/hyperactivité (E; } \\
\text { F; M) }\end{array}$ \\
\hline Niveau faible de détresse émotive (E) \\
\hline Niveau faible de timidité $(\mathrm{F})$ \\
\hline Niveau faible d'impulsivité (G) \\
\hline Niveau faible d'extraversion (G) \\
\hline Niveau faible de névrosisme $(G)$ \\
\hline Haute estime de soi (C) \\
\hline Compétences cognitives \\
\hline Intelligence $(\mathrm{N} ; \mathrm{M} ; \mathrm{Q})$ \\
\hline Conscience de soi et de ses actes (N) \\
\hline Capacités ou styles d'adaptation (N) \\
\hline Contrôle émotionnel ( 0 ) \\
\hline Maturité sociale $(Q)$ \\
\hline Énergie psychique (Q) \\
\hline Attitudes et aspirations \\
\hline Attitudes négatives envers la délinquance $(F ; 0)$ \\
\hline Croyances/attitudes prosociales (B; I) \\
\hline Attitude positive envers les interventions et l'autorité $(L ; P)$ \\
\hline Planification du futur (orientations et buts) $(\mathrm{J} ; \mathrm{T})$ \\
\hline Comportements \\
\hline Implication dans des comportements prosociaux $(\mathrm{P} ; \mathrm{T})$ \\
\hline Compétences dans des comportements prosociaux (M) \\
\hline Utilisation efficace des temps libres (L) \\
\hline Implication religieuse $(\mathrm{J})$ \\
\hline Implication dans les activités familiales (U) \\
\hline Faible niveau de délinquance (autre que des actes violents) $(\mathrm{H})$ \\
\hline
\end{tabular}


TABLEAU 3 (suite)

\begin{tabular}{|l|}
\hline \multicolumn{1}{|c|}{ Facteurs familiaux } \\
\hline Caractéristiques familiales \\
\hline Mère plus âgée $(\mathrm{F} ; \mathrm{O})$ \\
\hline Mère travaille à temps plein $(\mathrm{G})$ \\
\hline Bonne harmonie entre les parents $(\mathrm{U})$ \\
\hline Nombre restreint d'enfants dans la famille $(\mathrm{G})$ \\
\hline Lien d'attachement fort à la famille $(\mathrm{C} ; \mathrm{J})$ \\
\hline Condition du milieu de vie familiale $(0)$ \\
\hline Parents impliqués dans des activités conventionnelles $(\mathrm{C})$ \\
\hline Pratiques parentales \\
\hline Faible utilisation des punitions corporelles $(\mathrm{F} ; \mathrm{S} ; \mathrm{U})$ \\
\hline Discipline maternelle $(\mathrm{G})$ \\
\hline Soutien parental $(\mathrm{C})$ \\
\hline Intérêt dans l'éducation $(\mathrm{G})$ \\
\hline Désaccord pour les conduites antisociales $(\mathrm{J})$ \\
\hline Supervision effective $(\mathrm{F} ; \mathrm{K})$ \\
\hline Constance de la discipline parentale $(\mathrm{M})$ \\
\hline
\end{tabular}




\section{Les considérations méthodologiques}

\section{Les comportements prédits}

La première dimension abordée pour expliquer la disparité des facteurs de protection découverts concerne les comportements prédits. La nature, l'intensité, ainsi que la source de l'information sont susceptibles de moduler les facteurs qui sont identifiés. Au regard des travaux recensés, le spectre des conduites criminelles et antisociales est passablement large. Il est possible que les facteurs qui préviennent des conduites spécifiques (C, D, E, F, H, I, J, K, N, M, O, R), p. ex. des vols (F) ou des conduites violentes graves $(\mathrm{E}, \mathrm{H}, \mathrm{K}, \mathrm{O})$, diffèrent de ceux qui préviennent les manifestations délinquantes ou criminelles dans leur ensemble (A, B, G, L, P, Q, S, U). Les deux seules études qui comparent les facteurs de protection selon différents comportements délinquants, l'étude de Bushway, Krohn, Lizotte, Phillips et Schmidt (C) et celle de Lösel et Bender $(\mathrm{M})$, vont dans le même sens et montrent que les facteurs qui protègent contre certains comportements antisociaux ou violents varient passablement.

Dans le même ordre d'idées, le fait de considérer un comportement par sa présence ou son absence, p. ex. avoir ou ne pas avoir un dossier criminel à l'âge adulte, en opposition à une mesure qui saisit l'intensité des comportements négatifs, p. ex. le nombre de crimes commis, peut également expliquer les divergences entre les études retenues. La source sur laquelle se fondent les mesures peut aussi expliquer l'état des connaissances sur les facteurs de protection. En criminologie, plusieurs travaux montrent que les données officielles et les données autorapportées amènent des résultats qui diffèrent passablement (Thornberry \& Krohn, 2003). En ce qui concerne les études sélectionnées, les comportements étudiés se basent dans certains cas sur des données autorapportées (A, D, E, F, H, I, M, O, R, S), dans d'autres sur des données officielles $(B, G, P, Q, T)$ et même parfois sur les constatations d'une tierce partie (K, parents; $G$, agents de probation; et J, éducateurs). Une seule étude (J) combine à la fois des données officielles et des données autorapportées. Bien que la nature des actes prédits diffère et que le nombre de facteurs de protection considérés soit restreint, dans cette étude de Herrenkohl et ses collègues, on distingue une certaine cohérence entre les facteurs qui préviennent la violence (autorapportées) et la délinquance (dossier officiel). Des études supplémentaires sont nécessaires afin de déterminer l'impact réel des considérations entourant 
le choix des comportements prédits sur l'identification des facteurs de protection.

\section{La conceptualisation}

Au regard des études retenues, la définition liée aux facteurs de protection n'est pas en soi un point de discordance. De manière générale, les facteurs de protection sont définis comme des facteurs qui diminuent les probabilités des conduites criminelles et antisociales ultérieures (Lösel \& Bender, 2003; Pollard, Hawkins \& Artur, 1999; van Domburgh, Loeber, Bezemer, Stallings \& Stouhamer-Loeber, 2009 - U). Cette définition laisse toutefois en suspens certains éléments essentiels et plus complexes derrière le rôle ambivalent et l'effet de certains facteurs. Peu d'études retenues considèrent la variété des mécanismes et des processus par lesquels les facteurs de protection opèrent. Les facteurs de protection peuvent intervenir de deux manières, ils peuvent avoir un effet qui prévient l'engagement dans la délinquance de façon directe ou indirecte $(\mathrm{N})$. Les effets directs sont simples à interpréter, ils s'expriment par une association entre le facteur de protection et la variable prédite, la délinquance par exemple, dans laquelle le facteur diminue significativement les probabilités de délinquance. Pour illustrer ce type de relation, l'étude de Farrington et Ttofi (2011) (G) montre que dans un groupe de jeunes garçons turbulents, ceux qui affichent une faible extraversion, mesurée entre 8 et 10 ans, ont moins de chances d'être condamnés pour un crime à l'âge adulte.

Les effets indirects des facteurs de protection sont plus complexes, puisqu'ils interagissent avec les facteurs de risque. Les effets indirects des facteurs de protection se manifestent de deux manières, ils peuvent agir en tant que modérateur ou médiateur (A). Une variable médiatrice ou modératrice est une troisième variable qui change l'association entre le facteur de risque et la variable dépendante (Baron \& Kenny, 1986). Plus précisément, une variable modératrice affecte le lien entre deux variables alors qu'une variable médiatrice agit directement sur la variable dépendante (Baron \& Kenny, 1986; Barr, Boyce \& Zeltzer, 1996; Holmbeck, 1997; Little, Axford \& Morpeth, 2004). Autrement dit, les facteurs de protection qui jouent le rôle de variables modératrices modulent la nature (direction et/ou force) du lien entre le facteur de risque et la variable dépendante, alors que l'effet de variables médiatrices 
explique ou spécifie les circonstances qui accompagnent l'effet du facteur de risque.

Les variables modératrices et médiatrices fournissent des informations utiles sur comment, pourquoi, ou quand un phénomène se produit (Bennett, 2000). Elles peuvent ainsi permettre de comprendre comment s'opère l'effet des facteurs de protection, ce qui permet d'évaluer l'impact d'interventions consacrées à la neutralisation de facteurs de risque et d'orienter les programmes de prévention. Dans le numéro spécial de l'American Journal of Preventive Medecine, Lösel et Farrington (2012) soulignent l'importance des mécanismes indirects; toutefois, aucune des études présentées dans ce numéro ne met en évidence ces mécanismes. Parallèlement, deux études sélectionnées sont intéressées spécifiquement aux mécanismes indirects. Lösel et Bender (2014) (M) examinent les facteurs de protection qui ont le potentiel de modérer la relation entre l'intimidation (bullying) et les comportements antisociaux ou violents. Il ressort de cette recherche que plusieurs facteurs modèrent la relation qui vient d'être décrite, par exemple la constance de la discipline parentale, l'intelligence, une attitude positive envers l'école, pour ne nommer que ceux-là. La recherche de Stoddard et ses collègues (2013) montre quant à elle que l'accumulation de facteurs de protection modère la relation entre le cumul des facteurs de risque et les comportements violents. La confirmation ou l'identification des différents types de facteurs de protection dépend grandement de la conceptualisation initiale de la recherche.

Niveau de risque (les facteurs de risque considérés dans les modélisations)

Les probabilités d'un passage à l'acte délinquant ou antisocial sont grandement modulées par les facteurs de risque. Selon certains auteurs, le cumul de ces facteurs peut avoir un effet additif ou multiplicatif, voire exponentiel sur ces probabilités (Garmezy, 1985; Masten, Best \& Garmezy, 1990). Le lien entre les facteurs de protection et les facteurs de risque est donc important à considérer lors des analyses. Rappelons que les facteurs de protection peuvent agir de manière indirecte, en affaiblissant ou en neutralisant un facteur de risque. Le choix des facteurs de risque dans les modélisations est important, car ceux-ci ont le potentiel d'améliorer notre compréhension de la chaîne de médiation par laquelle les facteurs de protection opèrent. Farrington et ses 
collègues (2008) (F) confirment que le degré ou la dose de risque est un critère central dans l'étude des effets protecteurs. Or, les facteurs de risque considérés varient passablement entre les études retenues, ce qui représente une autre cause possible expliquant la disparité dans les facteurs de protection identifiés.

Comme le souligne Hall et ses collègues (2012a), en raison de l'importance du niveau de risque et des effets indirects des facteurs de protection, il devient important d'étendre le cadre des facteurs de protection non seulement en définissant de nouveaux facteurs, mais aussi en déterminant les différents moyens par lesquels les prédicteurs peuvent opérer et en documentant les répercussions de chaque opération sur les résultats.

\section{L'opérationnalisation}

La manière de mesurer et d'analyser les facteurs de protection est loin de faire l'unanimité, elle est même source d'un débat incessant dans les études étiologiques (Farrington, 1994; Wikstrom \& Loeber, 2000). En plus de la source des données qui est susceptible d'interférer, les choix dans l'opérationnalisation des facteurs de protection peuvent aussi être à l'origine de constats divergents entre les travaux empiriques.

Pour faciliter l'interprétation des résultats, mais aussi leur compréhension et leur portée, de nombreuses études choisissent de mesurer les facteurs de protection à l'aide de variables dichotomiques. Le principal inconvénient lié à cette manière d'opérationnaliser les facteurs de protection est la perte d'information qui en résulte. Le choix d'une telle mesure ne permet pas d'apprécier l'effet d'escalade d'une variable, les effets qui accompagnent chaque augmentation ou diminution, plus particulièrement, les cas en dessous du seuil sont considérés comme équivalents (Loeber \& Farrington, 2000).

De plus, ce choix opérationnel néglige l'ambivalence qui concerne certains facteurs qui sont à la fois des facteurs de risque et de protection. Par exemple, le rôle de la supervision des parents dans la délinquance peut être un facteur de risque (Jang \& Smith, 1997) ou un facteur de protection (Farrington et al., 2008 - F; Stouthamer-Loeber et al., 1993; van Domburgh et al., 2009 - U). Ainsi, dans certaines conditions, des facteurs peuvent accentuer les risques, alors que dans d'autres, ils procurent un effet préventif. Autrement dit, en utilisant des variables dichotomiques, plusieurs études négligent la dualité de certains facteurs 
et se concentrent uniquement sur l'une des deux facettes (positive ou négative). Une manière d'examiner les rôles est d'utiliser des variables trichotomiques, cette technique permet d'analyser si l'association entre le facteur et le comportement négatif est linéaire ou non, si le facteur agit exclusivement comme un facteur de risque ou de protection, ou encore s'il possède ces deux rôles (pour plus de détails, voir Loeber \& Farrington, 2012). Les études qui ont adopté ce type de mesure (E, F, $\mathrm{G}, \mathrm{H}, \mathrm{K}, \mathrm{O}, \mathrm{S}, \mathrm{U}$ ) montrent notamment que les facteurs traditionnellement considérés comme augmentant les risques peuvent aussi avoir un effet protecteur. Ces travaux mettent en évidence la pertinence et la nécessité de cet examen dans l'étude des facteurs de protection. Bien que cette manière d'examiner le rôle des facteurs ait ses mérites, elle n'est toutefois pas toujours disponible ou possible, puisqu'elle s'applique uniquement lorsque la variable est mesurée de façon continue ou ordinale. Puis, certains facteurs ne se prêtent pas à une telle opération, parce qu'ils sont par définition des mesures dichotomiques «naturelles», par exemple le fait de vivre avec ses deux parents biologiques, d'être victime de mauvais traitements, etc. $(\mathrm{O})$.

La décision d'examiner l'effet de facteurs spécifiques, ou plutôt l'impact du cumul de ces facteurs, est également sujet de débat. L'étude de Stoddard et al. (2013) montre bien l'effet modérateur du cumul des facteurs de protection, mais n'examine pas l'apport de facteurs spécifiques, ce qui ne permet pas d'apprécier ou de comparer les contributions. Bien qu'il y ait peu d'études sur cette question précise, les recherches recensées arrivent à des conclusions similaires, soit que le nombre de facteurs de protection possède une valeur prédictive ajoutée en comparaison des facteurs individuels $(\mathrm{A}, \mathrm{T})$. Stattin et ses collègues (1997) (Q) amènent par leur démonstration des nuances intéressantes dans leur étude sur la prédiction de la criminalité au sein de trajectoires adultes. Ils montrent notamment que l'effet du nombre est plus saillant pour les facteurs de risque (comportements à risque et caractéristiques individuelles antécédentes) que pour les facteurs de protection (compétences personnelles).

Bien qu'une telle opération augmente la valeur prédictive d'un modèle, celle-ci renseigne peu sur les effets particuliers des facteurs de protection, plus précisément à savoir comment ils interagissent avec les facteurs de risque. L'étude de Bushway et al. (2013) (C) propose une alternative intéressante en intégrant simultanément dans sa modélisation les facteurs uniques et le cumul des facteurs de protection par domaine 
(individuel, familial et social). La démonstration présentée dans cet article permet ainsi d'apprécier à la fois la contribution de facteurs spécifiques, mais aussi les domaines de facteurs les plus influents.

\section{Les caractéristiques des populations étudiées}

\section{Type d'échantillon}

Les facteurs de protection identifiés dépendent en grande partie des caractéristiques de l'échantillon auquel ils s'appliquent. Des travaux montrent que les individus qui développent une personnalité antisociale se démarquent par la précocité et la persistance des actes antisociaux qu'ils commettent (Hodgins, Côté \& Toupin, 1998; Loeber, 1990). Il est alors permis de croire que les facteurs qui vont prévenir les passages à l'acte antisocial au sein de trajectoires à risque ne seront pas les mêmes que ceux qui affectent les trajectoires des individus qui n'affichent pas de tels risques. Farrington et ses collègues (2008) (F) distinguent à la base deux types de facteurs, ceux-ci diffèrent en fonction de la population sur laquelle ils sont effectifs, alors que le premier type (preventive promotive factors) abaisse les probabilités de délinquance dans la population générale, le second (remedial promotive factors) prédit plutôt le désistement des activités criminelles au sein des populations délinquantes. Lors de deux démonstrations distinctes, Farrington et ses collègues $(2008,2011)$ (F; G) mettent en évidence des différences importantes entre les divers sous-groupes, notamment au regard des facteurs de protection effectifs. Par exemple, l'un des résultats saillants de l'étude de Farrington et Ttofi (2011) (G) montre qu'une bonne supervision parentale réduit la probabilité de récidive dans le groupe des garçons turbulents, cet effet préventif de la supervision ne s'applique toutefois pas dans le groupe composé des autres garçons. L'étude de Baglivio et al. (2014) (B), qui divise les jeunes selon les caractéristiques de leur délinquance, arrive à des conclusions similaires, à l'effet que les jeunes qui affichent une délinquance à la fois sévère, violente et chronique diffèrent significativement des autres jeunes quant à l'influence et à la nature des facteurs de protection qui les affectent. Ainsi, les facteurs de protection qui s'appliquent à la trajectoire de délinquance «commune» sont possiblement différents de ceux qui s'appliquent aux trajectoires marquées par des conduites délinquantes et antisociales plus intenses. Comme Stouthamer-Loeber et ses collègues (1993) le 
suggèrent, les facteurs de risque et de protection n'opèrent pas de manière uniforme selon l'intensité de la délinquance.

\section{Sexe des participants}

Au regard des études sélectionnées, bien que douze études basent leur démonstration sur des échantillons mixtes, seules cinq recherches examinent de manière spécifique les différences sexuelles, pour les sept autres études (B, C, E, H, J, K, N), le sexe des participants est utilisé exclusivement à titre de variable contrôle, cette caractéristique n'est d'ailleurs pas toujours présentée dans les tableaux de résultats. La plupart des études intéressées par l'effet de cette caractéristique individuelle trouvent peu $(\mathrm{A}, \mathrm{L})$, voire aucune différence entre les garçons et les filles (I, R). Une exception: les études de Bender et Lösel (1997) (D) et de Lösel et Bender (2003) (M) montrent que les facteurs de protection ne sont pas les mêmes selon le sexe des jeunes. On découvre que le réseau et le soutien social ont plus d'importance pour les filles comparativement aux garçons. Par exemple, ils observent que les filles sont généralement plus tributaires de l'influence sociale (D). En somme, Bender et Lösel (1997) (D) concluent que les relations entre les pairs et le soutien social peuvent avoir des effets différentiels selon le sexe, mais aussi en fonction de l'intensité des conduites antisociales.

En ce qui concerne les facteurs de risque, des travaux montrent que l'influence du réseau social sur les comportements antisociaux n'est pas la même pour les deux sexes (Caspi, Lynam, Moffitt \& Silva, 1993; Stattin \& Magnusson, 1990). La synthèse des connaissances effectuée par Javdani et ses collègues (2011) met bien en évidence le rôle des différences sexuelles dans la compréhension des conduites antisociales. Leurs analyses montrent que certains facteurs de risque, par exemple les abus sexuels durant l'enfance, ont une plus forte valeur explicative chez les femmes que chez les hommes. Ces chercheures concluent que le sexe est une variable écologique centrale dans l'explication des trajectoires antisociales. En l'absence d'une base empirique solide, il demeure important de tenir compte de l'hétérogénéité des jeunes à risque, ainsi que des différences sexuelles. 


\section{Stade de développement}

À l'instar du sexe des participants, on ne peut écarter la possibilité que les facteurs de protection dépendent également du stade de développement examiné, qu'ils varient en fonction de l'âge des participants. Encore une fois, peu d'études ont comparé les facteurs de protection selon les stades de développement. Soulignons à nouveau que les données nécessaires à cet exercice sont rares. L'étude de Farrington et ses collègues (2008) (F) porte une attention à cette comparaison. Ils montrent que les facteurs qui préviennent deux types de comportements délinquants, la violence et les vols, diffèrent selon le stade de développement. Par exemple, le peu de traits psychopathiques à l'enfance s'associe à de faibles probabilités de vols; en contrepartie, l'effet de ce facteur n'est toutefois pas significatif à l'adolescence. Les résultats de Bushway et ses collègues (2013) vont dans le même sens, on remarque que pour les mêmes comportements (violence et port d'arme), les facteurs diffèrent significativement entre l'adolescence et le début de l'âge adulte, que le travail et les relations amoureuses ont davantage d'impact sur les trajectoires une fois la majorité acquise.

\section{Conclusion}

L'étude des facteurs de protection liée aux conduites délinquantes et antisociales est encore un champ relativement nouveau et a-théorique. Par conséquent, l'absence de ligne directrice rend les comparaisons entre les études sur le sujet difficiles. En vertu des dimensions abordées dans cette synthèse des connaissances, on retient qu'il est difficile de tirer des conclusions quant aux facteurs spécifiques qui préviennent les conduites délinquantes et antisociales. De manière générale, cet état de choses s'explique notamment par la complexité des processus développementaux et la difficulté de reproduire avec fidélité les mesures déployées dans les études antérieures. Il ressort que les facteurs de protection identifiés dépendent notamment de multiples considérations méthodologiques, mais aussi des caractéristiques individuelles des participants.

Il nous importe de rappeler que les études sélectionnées ont été choisies en raison de la qualité de leur démonstration empirique. Nous croyons que chacune d'entre elles apporte une contribution significative. Toutefois, l'objectif sous-jacent à notre recherche était de faire la somme 
des apprentissages liés à ces travaux. Individuellement pertinente, la somme de ces recherches met en évidence des lacunes importantes dans l'étude des facteurs de protection.

Lorsque l'on évalue les résultats de notre synthèse des connaissances, force est de constater le grand nombre de facteurs aptes à prévenir la délinquance et les conduites antisociales. Il est possible que ce constat témoigne du caractère exploratoire qui entoure cet objet d'étude. La quasi-absence de validation des facteurs découverts en est la preuve. Les faibles bases empiriques sur lesquelles s'appuient nos connaissances sur les facteurs de protection s'expliquent, du moins en partie, par l'absence de cadre conceptuel et méthodologique solidement établi. Notamment, la définition opérationnelle de ces facteurs est loin de faire consensus. Par exemple, l'absence d'un facteur de risque peut être considérée comme un facteur de protection, les facteurs de risque et de protection peuvent aussi être vus comme les deux faces d'une même médaille $(F, G, U, S)$ alors que pour d'autres, les facteurs de protection représentent des construits indépendants qui réduisent directement ou indirectement la probabilité des conduites criminelles et antisociales (Rutter 1987; Werner \& Smith, 1992). En aval, ces multiples définitions opérationnelles influencent les facteurs qui seront découverts et limitent les comparaisons entre les études.

En ce sens, l'initiative du Centers for Disease Control and Prevention aux États-Unis est très instructive, en particulier en ce qui concerne les considérations méthodologiques entourant l'étude des facteurs de protection. L'idée de tester un même cadre conceptuel, à peu de différences près, en utilisant des données longitudinales prospectives provenant de quatre enquêtes différentes est novatrice. Soulignons en particulier la contribution du texte de Lösel et Farrington (2012) et celui de Loeber et Farrington (2012) dans la mise en place de ce cadre conceptuel et analytique. D'ailleurs, notre synthèse des connaissances s'accorde avec de nombreux éléments soulevés dans ce numéro spécial, à propos notamment de la conceptualisation et de l'opérationnalisation. On peut toutefois se questionner dans ces quatre études empiriques sur la valeur ou la pertinence de la recherche de facteurs qui préviennent des comportements antisociaux qui a été effectuée dans des populations générales (Rutter, 1985, 1987). Car on sait déjà que seule une petite proportion des individus qui affichent des risques élevés s'engagera et persistera dans la criminalité à l'âge adulte, que ce groupe sera responsable de la majorité des crimes commis dans nos sociétés. Il devient 
alors d'intérêt d'examiner de près ce groupe d'individus. Basée sur des critères précis, la synthèse des connaissances présentée dans cet article a mis en évidence l'importance du contexte des caractéristiques des populations étudiées. Le manque de considérations pour ces éléments explique possiblement la disparité des facteurs découverts dans les quatre études de ce numéro spécial de l'American Journal of Preventive Medecine.

En somme, la littérature sur les facteurs de protection n'est pas abondante et, dans presque tous les cas, elle ne réussit pas à identifier les facteurs de protection ou les mécanismes en tenant comptent des sous-groupes à risque, des différences sexuelles et celles liées au stade de développement. Autrement dit, les études précédentes n'ont pas été à même d'identifier l'impact des caractéristiques des populations étudiées, ce qui contribue à la méconnaissance des facteurs protecteurs qui s'appliquent à chaque sous-groupe d'enfants/adolescents à risque de s'engager dans la criminalité.

Dans cet article, nous avons donc exploré les connaissances sur les facteurs de protection plus en détail. Les dimensions abordées ont permis de soulever différents aspects qui méritent d'être considérés dans l'étude des facteurs de protection. En outre, à la lumière des écrits recensés, des considérations conceptuelles et méthodologiques sont proposées à titre de pistes de recherche. Nous identifions les pistes suivantes dans une optique constructive, pour poursuivre les efforts entamés dans l'étude des facteurs de protection:

1. Tenir compte que la population des enfants et des adolescents à risque de commettre des actes délinquants et antisociaux est hétérogène. De nombreux troubles sont associés à des risques accrus, par exemple les problèmes de conduite ou l'insensibilité affective;

2. Anticiper que différents facteurs sont susceptibles d'influencer la chaîne causale à différents stades du développement;

3. Vérifier les effets médiateurs potentiels sur l'ensemble des mécanismes causaux postulés;

4. Vérifier l'effet modérateur des facteurs de protection à chaque stade de développement, mais aussi en fonction des caractéristiques familiales et individuelles;

5. Examiner les hypothèses alternatives afin d'identifier la constellation des facteurs de protection qui change avec le temps et qui mène aux différents troubles (p. ex.: les différences sexuelles, le 
nombre de facteurs versus les facteurs spécifiques, les différences quant aux stades de développement).

Comme les études répertoriées dans cette synthèse des connaissances ont été réalisées a posteriori, sans base théorique initiale, il serait recommandé de développer un programme d'étude avec un objectif de validation des facteurs de protection déjà établis. De concert avec une collecte de données rendant possible l'examen de différents choix méthodologiques, permettant ainsi de statuer sur l'impact de ces décisions sur les résultats. À la suite de la synthèse des connaissances présentée, les éléments suivants sont recommandés : échantillonner auprès d'une population très hétérogène pour ainsi tester l'effet de différentes caractéristiques des populations (p. ex. : jeune, adulte, homme, femme, à faible risque, sous-groupes à haut risque, etc.), se baser sur un devis longitudinal afin d'examiner les facteurs de protection dans le temps, bénéficier d'un large éventail de comportements à prédire et mesurer les facteurs de protection de manière précise afin de pouvoir modifier leur modélisation, mais aussi pour tester différents types d'effets. Finalement, l'étude des facteurs de risque, comme il a été exprimé précédemment, pourrait également permettre d'enrichir ces propositions et de suggérer des directives plus claires pour de futures recherches.

Les résultats de cette synthèse se doivent d'être tempérés. Notamment, en raison du nombre limité de recherches disponibles aux fins d'examen $(\mathrm{N}=21$ études). Par le fait même, cette synthèse nous a tout de même permis de constater l'état rudimentaire des connaissances sur les facteurs de protection. Il est primordial de poursuivre la recherche sur ces facteurs, car leurs retombées potentielles sur les pratiques sont importantes, ils pourraient notamment servir à orienter les programmes de prévention dès l'enfance et à formuler des réponses adaptées aux enfants et aux adolescents à risque.

\section{Références}

Arthur, M. W., Hawkins, D., Pollard, J. A., Catalano, R. F., \& Baglioni, A. J. (2002). Measuring Risk and Protective Factors for Use, Delinquency, and Other Adolescent Problem Behaviors: The Communities That Care Youth Survey. Evaluation Review, 26(6), 575-601.

Baglivio, M. T., Jackowski, K., Greenwald, M. A., \& Howell, J. C. (2014). Serious, Violent, and Chronic Juvenile Offenders. Criminology \& Public Policy, 13(1), 83-116. 
Baron, R., \& Kenny, D. (1986). The moderator-mediator variable distinction in social psychological research. Journal of Personality and Social Psychology, 51(6), 1173-1182.

Barr, R. G., Boyce, W. T., \& Zeltner, L. K. (1996). The stress-illness association in children: a perspective from the biobehavioural interface. Dans R. J. Haggerty, L. R. Sherrod, N. Garmezy \& M. Rutter (Éds), Stress, Risk and Resilience in Children and Adolescents: Processes, Mechanisms, and Intervention (pp.182-224). Cambridge: Cambridge University Press.

Bender, D., \& Lösel, F. (1997). Protective and risk effects of peer relations and social support on antisocial behaviour in adolescents from multi-problem milieus. Journal of adolescence, 20(6), 661-678.

Bennett, J. A. (2000). Focus on Research Methods Mediator and Moderator Variables in Nursing Research: Conceptual and Statistical Differences. Research in Nursing $\mathcal{F}$ Health, 23(5), 415-420.

Bernat, D., Oakes, J. M., Pettingell, S. L., \& Resnick, M. (2012). Risk and direct protective factors for youth violence: results from the national longitudinal study of adolescent health. American Journal of Preventive Medicine, 43(2S1, S57-S66).

Blumstein, A., Cohen, J., \& Farrington, D. P. (1988). Criminal Career Research: It's Value for Criminology. Criminology, 2(6), 1-35.

Bushway, S. D., Krohn, M. D., Lizotte, A. J., Phillips, M. D., \& Schmidt, N. M. (2013). Are risky youth less protectable as they age? The dynamics of protection during adolescence and young adulthood. Justice quarterly, 30(1), 84-116.

Caspi, A., Lynam, D., Moffitt, T. E., \& Silva, P. A. (1993). Unraveling girls delinquency: Biological, dispositional, and contextual contributions to adolescent misbehaviour. Developmental Psychology, 29(1), 19-30.

Chaiken, M. R., \& Chaiken, J. M. (1985). Who gets caught doing crime? Washington, D.C: U. S, Department of Justice, Bureau of Justice Statistics.

Farrington, D. P. (1994). Interactions between individual and contextual factors in the development of offending. Dans R. K Silbereisen \& E. Todt (Éds.), Adolescence in Context: The Interplay of Family, School, Peers and Work in Adjustment (pp.366-381). New York, N. Y: Springer-Verlag.

Farrington, D. P. (2000). Explaining and preventing crime: The globalization of knowledge - The American Society of Criminology 1999 Presidential Address. Criminology, 38(1), 1-24.

Farrington, D. P., Loeber, R., Jolliffe, D., \& Pardini, D. A. (2008). Promotive and Risk Process at Different Life Stages. Dans R. Loeber, D. P. Farrington, M. Stouthamer-Loeber \& H. Raskin White (Éds.), Violence and Serious Theft: Development and Prediction from Childhood to Adulthood (pp.169-230). London: Routledge.

Farrington, D. P., \& Ttofi, M. M. (2011). Protective and Promotive Factors in the Development of Offending. Dans T. Bliesener, A. Beelmann \& M. Stemmler (Eds), Antisocial behaviour and crime: Contributions of developmental and evaluation research to prevention and intervention (pp.71-88). Cambridge: Hogrefe Publishing. 
Farrington, D. P., \& Welsh, B. (2007). Saving children from a life of crime: Early risk factors and effective interventions. Oxford: University Press.

Garmezy, N. (1985). Stress resistant children: The search for protective factors. Dans Recent Research in Developmental Psychopathology. Journal of Child Psychology and Psychiatry, 34(4), 213-233.

Hall, J. E., Simon, R. T., Lee, R. D., \& Mercy, J. A. (2012a). Implications of direct protective factors for public health research and prevention strategies to reduce youth violence. American Journal of Preventive Medecine, 43(2S1, S76-S84).

Hall, J. E., Simon, T. R., Mercy, J. A, Loeber, R., Farrington, D. P. \& Lee R. D. (2012b). Centers for Disease Control and Prevention's Expert Panel on Protective Factors for Youth Violence Perpetration: Background and Overview. American Journal of Preventive Medicine, 43(2S1, S1-S7).

Henry, D. B, Tolan, P. H., Gorman-Smith, D., \& Schoeny, M. E. (2012). Risk and direct protective factors for youth violence: results from the CDC multisite violence prevention project. American Journal of Preventive Medecine, 43(2S1, S67-S75).

Herrenkohl, T. I., Hill, K. G., Chung, I.-J., Guo, J., Abbott, R. D., \& Hawkins, J. D. (2003). Protective factors against serious violent behaviour in adolescence: A prospective study of aggressive children. Social Work Research, 27(3), 179-191.

Herrenkohl, T. I., Lee, J., \& Hawkins, J.D. (2012). Risk versus direct protective factors and youth violence in the Seattle social development project. American Journal of Preventive Medicine, 43(2S1, S41-S56).

Herrenkohl, T. I., Tajima, E. A., Whitney, S. D., \& Huang, B. (2005). Protection against antisocial behaviour in children exposed to physically abusive discipline. Journal of Adolescent Health, 36, 457-465.

Hodgins, S., Côté, G., \& Toupin, J. (1998). Major mental disorder and crime: an etiological hypothesis. Dans D. Cooke, A. Forth \& R. D. Hare (Eds.), Psychopathy: Theory, Research, and Implications for Society (pp.231-256). Dordrecht: Kluwer Academic Publisher.

Hoge, R. D., \& Leshied, A. W. (1996). An Investigation of Risk and Protective Factors in a Sample of Youthful Offenders. Journal of Child Psychology and Psychiatry, 37(4), 419-424.

Holmbeck, G. N. (1997). Toward terminological, conceptual, and statistical clarity in the study of mediators and moderators: examples from the ChildClinical and Pediatric Psychology literatures. Journal of Counselling and Clinical Psychology, 65(4), 599-610.

Hurrelmann, K., \& Lösel, F. (1990). Health Hazards in Adolescence. Berlin/New York: De Gruyter.

Jang, S., \& Smith, C. A. (1997). A test of reciprocal causal relationships among parental supervision, affective ties, and delinquency. Journal of Research in Crime and Delinquency, 34, 307-336. 
Javdani, S., Sadeh, N., \& Verona, E. (2011). Expanding our lens: Female pathways to antisocial behavior in adolescence and adulthood. Clinical Psychology Review, 31, 1324-1348.

Kratzer, L. \& Hodgins, S. (1999). A typology of offenders: A test of Moffitt's theory among males and females from childhood to age 30. Criminal Bebaviour $\mathcal{E}$ Mental Health, 9, 57-73.

Laplante, B., Street, M. C., \& Santillan, M. M. (2006, novembre). L'analyse biographique des données d'enquêtes longitudinales prospectives à période d'observation courte. Communication présentée à la Chaire Quételet. Les systèmes d'information en démographie et en sciences sociales. Nouvelles questions, nouveaux outils? Louvain-la-Neuve.

Little, M., Axford, N., \& Morpeth, L. (2004). Research review: risk and protection in the context of services for children in need. Child and Family Social Work, 9, 105-117.

Loeber, R. (1990). Development and risk factors of juvenile antisocial behavior and delinquency. Clinical Psychology Review, 10, 1-41.

Loeber, R., \& Farrington, D .P. (2000). Young children who commit crime: Epidemiology, developmental origins, risk factors, early interventions, and policy implications. Development and Psychopathology, 12, 737-762.

Loeber, R., \& Farrington, D. P. (2012). Advancing knowledge about direct protective factors that may reduce youth violence. American Journal of Preventive Medicine, 43(2S1, S24-S27).

Lösel, F., \& Bender, D. (2003). Protective factors and resilience. Dans D. P. Farrington \& J. W. Coid (Éds), Early Prevention of Adult Antisocial Behaviour (pp. 130-140). Cambridge: University Press.

Lösel, F., \& Bender, D. (2014). Online first, Aggressive, Delinquent, and Violent Outcomes of School Bullying: Do Family and Individual Factors Have a Protective Function? Journal of School Violence, 13(1), 59-79.

Lösel, F., \& Blisener, T. (1994). Some High-risk Adolescents do not Develop Conduct Problems: A Study of Protective Factors. International Journal of Behavioral Development, 17(4), 753-777.

Lösel, F., \& Farrington, D. P. (2012). Direct protective and buffering protective factors in the development of youth violence. American Journal of Preventive Medicine, 43(2S1, S8-S23).

Masten, A. S., Best, K. M. \& Garmezy, N. (1990). Resilience and development: Contributions from the study of children who overcome adversity. Development and Psychopathology, 2, 425-444.

Masten, A. S., \& Garmezy, N. (1985). Risk, vulnerability and protective factors in developmental psychopathology. Dans B. B Lahey \& A. E Kazdin (Éds.), Advances in clinical child psychology (pp. 1-52). New York, NY: Plenum.

Moffitt, T. E., Caspi, A., Harrington, H., \& Milne, B. J. (2002). Males on the life-course-persistent and adolescence-limited antisocial pathways: followup at age 26 years. Development and Psychopatbology, 14(1), 179-207. 
Pardini, D. A., Loeber, R., Farrington, D. P., \& Stouthamer-Loeber, M. (2012). Identifying direct protective factors for nonviolence. American Journal of Preventive Medicine, 43(2S1, S28-S40).

Piquero, A. R. (2000). Are Chronic Offenders the Most Serious Offenders? Exploring the Relationship with Special Attention to Offense Skewness and Gender Differences. Journal of Criminal Justice, 28, 103-116.

Pollard, J. A., Hawkins, J. D., \& Arthur, M. W. (1999). Risk and protection: Are both necessary to understand diverse behavioral outcomes in adolescence? Social Work Research, 23(8), 145-58.

Pratt, T. C. (2002). Meta-Analysis and Its Discontents: Treatment Destruction Techniques Revisited. Journal of Offender Rehabilitation, 35(1), 23-40.

Pulkkinen, L. (1998). Levels of longitudinal data differing in complexity and the study of continuity in personality characteristics. Dans R. B. Cairns, L.R. Bergman \& J. Kagan (Éds.), Methods and models for studying the individual (pp.161-183). Thousand Oaks, CA: Sage Publications.

Rennie, C., \& Dolan, M. (2010). The signifiance of protective factors in the assessment of risk. Criminal Behavior and Mental Health, 20, 8-22.

Rosenthal, R., Dimatteo, M. R. (2001). Meta-analysis: recent developments in quantitative methods for literature reviews. Annual Review of Psychology, 52, 59-82.

Rutter, M. (1985). Resilience in the face of adversity. Protective factors and resistance to psychiatric disorder. The British Journal of Psychiatry, 147, 598611.

Rutter, M. (1987). Psychosocial Resilience and Protective Mechanisms, American Journal of Orthopsychiatry, 57(3), 316-331.

Stattin, H., \& Magnusson, D. (1990). Pubertal maturation in female development. Vol. 2 in the series Paths through life. Hillsdale, NJ: Erlbaum.

Stattin, H., Romelsjö, A., \& Stenbacka, M. (1997). Personal ressources as modifiers of the risk for future criminality: An Analysis of Protective Factors in Relation to 18-Year-Old Boys. British Journal of Criminology, 37(2), 198223.

Stoddard, S. A., Whiteside, L., Zimmerman, M. A., Cunningham, R. M., Chermack, S. T., \& Walton, M. A. (2013). The relationship between cumulative risk and promotive factors and violent behavior among urban adolescents. American journal of community psychology, 51(1-2), 57-65.

Stouthamer-Loeber, M., Loeber, R., Farrington, D. P., Zhang, Q., Van kammen, W., \& Maguin, E. (1993). The double edge of protective and risk factors for delinquency: Interrelations and developmental patterns. Development and Psychopathology, 5, 683-701.

Stouthamer-Loeber, M., Wei, E., Loeber, R., \& Masten, A. S. (2004). Desistance from persistent serious delinquency in transition to adulthood. Development and Psychology, 16, 897-918.

Taylor, J. T., Friday, P. C., Ren, X., Weitekamp, E. G. M., \& Kerner, H.-J. (2004). Risk and Protective Factors Related to Offending: Results from a Chinese 
Cohort Study. The Australian and New Zealand Journal of Criminology, 37, 13-31.

Thornberry, T. P., \& Krohn, M. D. (2003). The Development of Panel Studies of Delinquency. Dans T. P. Thornberry \& M. D. Krohn (Éds.), Taking Stock of Delinquency: An Overview of Findings from Contemporary Longitudinal Studies (pp.1-9). New York, NY: Kluwer Academic/Plenum Publishers.

Van Domburgh, L., Loeber, R., Bezemer, D., Stallings, R., \& StouthamerLoeber, M. (2009). Childhood Predictors of Desistance and Level of Persistence in Offending in Early Onset Offenders. Journal of Abnormal Child Psychology, 37, 967-980.

Werner, E. E., \& Smith, R. S. (1992). Overcoming the Odds: High Risk Children from Birth to Adulthood. London: Cornell University Press.

Wikström, P. O. H. (1985). Everyday Violence in Contemporary Sweden. Stockholm: National Council for Crime Prevention.

Wikström, P. O. H., \& Loeber, R. (2000). Do disadvantaged neighborhoods cause well-adjusted children to become adolescent delinquents? Criminology, 38, 1109-1142.

Wolfgang, M., Figlio, R. M., \& Sellin, T. (1972). Delinquency in a Birth Cohort. Chicago, IL: University of Chicago Press.

\begin{abstract}
Most children and adolescents who engage in antisocial behaviour and delinquency do not continue to display these behaviours in adulthood. Therefore, there are factors in the natural environment that protect children-at-risk from becoming adult offenders. However, few studies have examined protective factors and the conceptual and methodological foundations for studying protective factors remain poorly developed. This article presents a review of empirical studies of factors that prevent antisocial and criminal behavior and the conceptual, limits and methodological issues that determine what factors are identified. Results show that a large number of protective factors have been identified. The lack of consistency in the findings reflects the exploratory nature of the studies and the virtual absence of replication studies. Among the methodological issues addressed, the importance of population characteristics is highlighted. Based on findings from the review, implications for future studies of protective factors are proposed, including the importance of taking account of the heterogeneity of children and adolescents at risk to offend in adulthood. Knowledge of protective factors is needed to inform policy decisions regarding investment in early childhood programs aimed at preventing criminal behaviour.
\end{abstract}

KEYWORDS • Protective factors, delinquency, antisocial behaviour, knowledge synthesis.

RESUMEN - La mayoría de los niños y adolescentes que cometen actos delictivos no persisten en la criminalidad en la edad adulta. Así, concebimos que existen en al ambiente inmediato, factores que protegen a los niños y a los adolescentes en riesgo. Ahora bien, la literatura sobre los factores de protección presenta lagunas; en especial, escasos estudios nos informan sobre los fundamentos conceptuales y metodológicos que encuadran el estudio de dichos factores. El presente artículo sintetiza los conocimientos, basándose en los trabajos empíricos sobre los factores de protección asociados a los comportamientos antisociales y criminales, y propone realizar un examen de las 
consideraciones conceptuales, metodológicas, y de los límites de los estudios seleccionados, susceptibles de influenciar los factores descubiertos. Los resultados muestran la identificación de un gran número de factores de protección. La falta de uniformidad en los resultados refleja el carácter exploratorio de los estudios, pero también, prácticamente la ausencia de validación de los factores descubiertos. Entre las cuestiones metodológicas abordadas, la importancia de las características de la población es puesta en evidencia. Basados en nuestros resultados, proponemos pistas de investigación, especialmente, de considerar la heterogeneidad de las poblaciones que presentan riesgos elevados de criminalidad a la edad adulta. Los conocimientos sobre los factores de protección son necesarios para esclarecer las decisiones políticas que conciernen la inversión en programas que apuntan a prevenir la criminalidad desde la temprana infancia.

PALABRAS CLAVE - Factores de protección, delincuencia, conductas antisociales, sintesis de conocimientos. 